\title{
Perfil anímico en pacientes con enfermedades crónicas: su relación con variables sociodemográficas y clínicas
}

\author{
Joel Martínez-Soto ${ }^{1}$ y Victor Manuel Ramos-Frausto ${ }^{2}$ \\ Universidad de Guanajuato-México
}

El objetivo del presente estudio es documentar el perfil anímico e índices de perturbación emocional (IPE) en pacientes adultos con cáncer, insuficiencia renal (IR), diabetes y enfermedad cardiovascular (ECV). Participaron 386 pacientes (cáncer $n=96$; IR $n=107$; diabetes $n=91$; ECV $n=92$ ) quienes autoreportaron sus estados de anímo negativos y positivos. Los resultados muestran que el perfil anímico de los pacientes con cáncer e IR es similar en cuanto a su caracterización y cuantificación, con IPE significativamente menores a los de los pacientes con diabetes y ECV. Por otra parte, comparados con demás grupos, los pacientes con ECV presentan puntajes más altos en todas las dimensiones negativas y positivas de los estados de anímo. Se analiza y discute la influencia de diversas variables sociodemograficas y clínicas en la adaptación y respuesta psicólogica de los pacientes.

Palabras clave: estados del humor, enfermedades crónicas, perturbación emocional, bienestar, psicologia de la salud

\section{Mood profile in patients with chronic diseases: its relationship with sociodemographic and clinical variables}

The objective of the present study is to document the mood profile and emotional disturbance indexes (EDI) in adult patients with cancer, renal insufficiency (RI), diabetes (D) and cardiovascular disease (CD). A total of 386 patients participated (cancer $n=96$; RI $\mathrm{n}=107$; diabetes $\mathrm{n}=91$; CD $\mathrm{n}=92$ ) who self-reported their negative and positive mood states. The results evidence that the mood profile of the patients with CA and IR is similar in terms of their characterization and quantification, with IPE significantly lower than that of the patients with $\mathrm{D}$ and CD. On the other hand, compared with other groups, patients with CD present higher scores in all negative and positive dimensions of mood states. The influence of various sociodemographic and clinical variables on the adaptation and psychological response of patients is analyzed and discussed.

Keywords: mood states, chronic disease, mood disturbance, well-being, health psychology

1 Doctor en Psicología. Profesor e investigador de tiempo completo, Departamento de Psicología, Universidad de Guanajuato, México. Dirección postal: Blvd. Puente Milenio No. 1001. Fracción del Predio San Carlos. C.P. 37670; León, Guanajuato, México. Contacto: masjmx@yahoo.com.mx https://orcid.org/0000-0002-9418-9726

2 Doctor en Salud Pública. Profesor e investigador de tiempo completo en el Departamento de Enfermería y Obstetricia, Universidad de Guanajuato, México. Dirección postal: Circuito del Bosque sur 461. Contacto: ramos.victor54@gmail.com https://orcid. org/0000-0001-9815-4777 


\section{Perfil de humor em pacientes com doenças crônicas: sua relaçáo com variáveis socio- demográficas e clínicas}

O objetivo do presente estudo é documentar o perfil de humor e os índices de distúrbios emocionais (EIP) em pacientes adultos com câncer, insuficiência renal (RI), diabetes e doenças cardiovasculares (DCV). Participaram 386 pacientes (câncer $n=96$; RI $n=107$; dibetes $\mathrm{n}=91$; DCV $\mathrm{n}=92$ ) que relataram seus estados de humor negativo e positivo. Os resultados mostram que o perfil de humor de pacientes com câncer e RI é semelhante em termos de caracterização e quantificação, com EIPs significativamente menores do que os de pacientes com diabetes e DCV. Por outro lado, em comparação com outros grupos, os pacientes com DCV apresentam escores mais altos em todas as dimensōes positivas e negativas dos estados de humor. A influência de várias variáveis sociodemográficas e clínicas na adaptação e resposta psicológica dos pacientes é analisada e discutida.

Palavras-chave: estados de humor, doenças crônicas, distúrbios emocionais, bem-estar, psicologia da saúde

\section{Profil d'humeur chez les patients atteints de maladies chroniques: sa relation avec les variables sociodémographiques et cliniques}

L'objectif de la présente étude est de documenter le profil de l'humeur et les indices de perturbation émotionnelle (IPE) chez les patients adultes atteints de cancer, d'insuffisance rénale (IR), de diabète et de maladies cardiovasculaires (MCV). 386 patients ont participé (cancer $\mathrm{n}=96$; $\mathrm{IR} \mathrm{n}=107$; dibetes $\mathrm{n}=91$; $\mathrm{CVD} \mathrm{n}=92$ ) qui ont auto-déclaré leurs états d'humeur négatifs et positifs. Les résultats montrent que le profil d'humeur des patients atteints de cancer et d'IR est similaire en termes de caractérisation et de quantification, avec des IPE significativement inférieurs à ceux des patients atteints de diabète et de MCV. D'un autre côté, par rapport aux autres groupes, les patients atteints de MCV ont des scores plus élevés dans toutes les dimensions négatives et positives des états d'humeur. L'influence de diverses variables sociodémographiques et cliniques sur l'adaptation et la réponse psychologique des patients est analysée et discutée.

Mots-clés: états d'humeur, maladies chroniques, troubles émotionnels, bien-être, psychologie de la santé 
Los cambios drásticos en la vida cotidiana acarreados desde el siglo pasado están alimentando la carga creciente de enfermedades crónicas no transmisibles (ECnT en adelante) (Smith, 2002). Dichas enfermedades son causa de la muerte de 41 millones de personas a nivel mundial cada año (OMS, 2018). Comparada con población saludable, las personas con ECnT son significativamente más propensas a padecer de afectaciones psicológicas que aquellas sin dicho trastorno, incrementando con ello la progresión de la enfermedad y sus repercusiones sociales y económicas (Andreoulakis et al., 2012).

La investigación sobre el estudio del perfil anímico de los pacientes con algún tipo de enfermedad crónica suele hacerse de forma aislada, focalizándose en un diagnóstico particular y con una tendencia a evaluar los efectos psicoafectivos para dicho diagnóstico, resultando con ello patrones emocionales de respuestas específicas para cada subespecialidad (Penninx et al., 1996). Actualmente son pocos los estudios multigrupo que den cuenta de las convergencias y divergencias de diferentes ECnT considerando el grado de perturbación emocional y estados anímicos. Se ha sugerido que el perfil anímico de los pacientes con ECnT puede ser independiente a su condición y reflejar tanto rasgos de personalidad como capacidades (Arpin et al., 1990). Otras investigaciones refieren diferencias pronunciadas en los estados anímicos en función del tipo de ECnT (Koenig et al., 1988; Palinkas, Wingard, y Barrett-Connor, 1990). Dentro de estos estudios también se considera importante añadir la influencia de los antecedentes sociodemográficos y clínicos de la propia enfermedad (Helgeson y Zajdel, 2017).

En la presente investigación se pretende abordar el estudio del perfil anímico y sus correlatos clínicos y sociodemográficos considerando cuatro submuestras de pacientes con ECnT: cáncer, insuficiencia renal (IR en adelante), diabetes y enfermedad cardiovascular (ECV en 
adelante). El abordaje psicológico de la caracterización del perfil anímico de dichos pacientes se hará con la escala de Perfil de Estados del Humor (Profile of Mood States por su acrónimo en ingles, POMS en adelante; McNair, Lorr, y Doppleman, 1971), la cual es una escala de mayor uso en el ámbito psicológico de evaluación clínica de los estados de ánimo en pacientes con ECnT. La escala evalúa seis dimensiones anímicas clínicamente relevantes: tensión, depresión, ira, vigor, fatiga y confusión (McNair, Lorr, y Droppleman, 1981). Mediante estas dimensiones es posible obtener un puntaje de malestar emocional referido como Índice de Perturbación Emocional (IPE en adelante), el cual es un índice general de alteración del estado de ánimo que se relaciona confiablemente con los factores de la escala POMS (McNair, Lorr, y Droppelman, 1992).

\section{Perfil anímico en pacientes con cáncer}

Desde un punto de vista psicológico, el perfil anímico establecido con la escala POMS en pacientes con cáncer puede caracterizarse por bajas puntuaciones en la escala de ira (Barinková y Mesároová, 2013) y de manera consistente de altas puntuaciones en las dimensiones de ansiedad, confusión y depresión (Koizumi et al., 2018; Groarke et al., 2018, Jiang et al., 2018). Los pacientes con cáncer que experimentan dolor crónico reportan mayores niveles de ansiedad, depresión, ira, fatiga y confusión, así como bajos niveles de vigor respecto a aquellos pacientes libres de dolor (Lin, Lai, y Ward, 2003).

Muestras de sobrevivientes, al compararse con población general, refieren valores promedio inferiores en la dimensión de vigor (WulffBurchfield et al., 2019). Respecto al contraste con otras enfermedades crónicas, los pacientes con cáncer de pulmón tienden a experimentar mayores perturbaciones emocionales que los pacientes con ECV (McCorkle y Quint-Benoliel, 1983). En términos de antecedentes clínicos, se reportan fluctuaciones en los estados de ánimo dependientes de la etapa de la enfermedad, tratamiento y diagnóstico (McCorkle y Quint-Benoliel, 1983). Los pacientes con cáncer que acuden a quimioterapia por lo general reportan tener altos niveles de fatiga y depresión 
(Kim, Hickok, y Morrow, 2006). Diferencias significativas en los niveles de ansiedad, depresión e ira pueden manifestarse con respecto a la ubicación del cáncer en el cuerpo (Allart-Vorelli et al., 2015).

En cuanto a la incidencia de antecedentes sociodemográficos en la expresión emocional de la enfermedad crónica, algunos estudios refieren diferencias por sexo y edad en cuanto a la vivencia de los estados anímicos, particularmente en cuanto a los niveles de fatiga, siendo las mujeres quienes de manera consistente reportan mayores niveles de fatiga al compararse con los varones (Annunziata et al., 2013). Con relación a la edad, son los más jóvenes quienes tienden a presentar mayores niveles de fatiga y depresión (Annunziata et al., 2013). Zabora et al. (2001) señalan que menores niveles de tensión y ansiedad pueden experimentarse respecto a la enfermedad en edades entre 30 y 60 ańos, con un incremento significativo en los niveles de perturbación emocional a partir de los 80 ańos. Akechi et al. (1999) documentan la influencia de los niveles de escolaridad y empleo en los niveles de fatiga percibida de pacientes con cáncer. Finalmente, Annunziata et al. (2013) documentan que, a mayor educación, mayor es la fatiga y depresión en pacientes oncológicos.

\section{Perfil animico en pacientes con IR}

En contraste con los pacientes con cáncer, poco se conoce sobre la caracterización del perfil anímico de los pacientes con IR. En términos generales, se refiere que tanto la fatiga como la depresión son dos de los problemas más recurrentes en este tipo de pacientes (Nowak y Laudański, 2014). Para dichos pacientes el nivel de ansiedad es un predictor independiente de la sintomatología somática, llegando a explicar el 25\% de la variancia total de síntomas informados (Perales-Montilla, Duschek, y Reyes del Paso, 2013).

Diversas de las alteraciones emocionales que suelen experimentar este tipo de pacientes tienen que ver con diferentes perdidas (de la función renal, bienestar, de su lugar en la familia y en el trabajo, de recursos financieros y de su función sexual), incertidumbre (supervivencia, dependencia de la tecnología) y el estrés inherente a los tratamientos 
(sesiones de diálisis, restricciones dietéticas) (Peralta-Montilla, GarcíaLeón, y Reyes, 2012).

En términos de antecedentes clínicos, mientras se sugiere que no existen diferencias significativas en cuanto al tipo de tratamiento recibido y los niveles de ansiedad y depresión (Fındıklı et al., 2016), también se refiere la existencia de diferencias en los niveles de depresión y fatiga en función del tipo de diálisis recibida, con una mayor prevalencia de depresión y fatiga en pacientes que reciben tratamiento de hemodiálisis (Rodrigue et al., 2010).

Por otra parte, una mayor prevalencia de estados anímicos subjetivos de fatiga puede ser dependiente del grado de avance de la enfermedad (Joshwa y Campbell, 2017), con una mayor prevalencia en etapas más avanzados del trastorno (Giordano et al., 2007). Asimismo, a mayor cantidad de meses de tratamiento con hemodiálisis, menor ansiedad (Paez et al., 2009). Pacientes quienes tuvieron un transplante de riñón, tienden a mostrar estados de ánimo (evaluados con la escala POMS) promedio a los de la población general (Tamura et al., 2018).

En comparación con otras enfermedades crónicas, Rodrigue et al. (2010) refieren que los niveles de fatiga en pacientes con IR son mayores a los reportados en pacientes con cáncer de mama. En cuanto a los antecedentes sociodemográficos, mayores niveles de depresión, fatiga y ansiedad están asociados con una mayor edad y un bajo nivel educativo (Keskin y Engin, 2011). En población latinoamericana, jóvenes de 17 a 36 ańos en tratamiento de hemodiálisis tienden a padecer una mayor recurrencia de trastornos depresivos (Atencio et al., 2004). Se han reportado también mayores niveles de fatiga asociados con el estatus marital, donde son las personas casadas quienes tienden a experimentar mayores niveles de fatiga (Williams, Crane, y Kring, 2007). En pacientes con hemodiálisis, el hecho de ser desempleado influye significativamente en altos niveles de fatiga emocional, depresión y ansiedad (Nowak y Laudański, 2014). Como otras enfermedades crónicas, son las mujeres quienes reportan mayores niveles de fatiga al compararse con los varones (Artom et al., 2014). 


\section{Perfil animico en pacientes con diabetes}

$\mathrm{Al}$ igual que los pacientes con IR, poco se conoce también sobre el perfil anímico de los pacientes con diabetes. Estudios refieren que las personas con diabetes no diagnosticada tienen un bajo riesgo de desarrollar trastornos afectivos comparadas con quienes si la tienen (Nouwen et al., 2011) y entre quienes son diagnosticados, el riesgo de presentar trastornos afectivos se incrementa después de dos años del diagnóstico (González-Zacarias et al., 2016).

En general la diabetes se asocia con un incremento del riesgo de depresión y la depresión se asocia con un incremento en el riesgo de padecer diabetes (Rotella y Mannucci, 2013). La prevalencia de la depresión en adultos con diabetes tipo 2 es aproximadamente el doble de alta comparada con aquellos adultos sin diabetes (Shin et al., 2017). En cuanto a la caracterización con la escala POMS, algunos estudios refieren que los pacientes diabéticos tienden a presentar mayores IPE al compararse con adultos saludables (Baptista, Machado-Rodríguez, y Martins, 2017; Breymeyer et al., 2016). En términos clínicos, una mayor cantidad de complicaciones relacionadas con la diabetes se asocia con una mayor presencia de depresión (De Groot et al., 2001). A la fecha no se cuentan con estudios que documenten la existencia de IPE basados en las características sociodemográficas de los pacientes con diabetes.

\section{Perfil anímico en pacientes con ECV}

El impacto psicológico de las enfermedades cardiacas en cuanto a los estados de ánimo, refieren la existencia de altos niveles de ansiedad (Bennett et al., 2002), depresión (Mbakwem, Aina, y Amadi, 2016), ira (Elliott et al., 2010) fatiga (Fink, et al., 2010) y comparados con la población general, bajos niveles de vigor (Fink et al., 2009). Al igual que la diabetes, se estima que los niveles de depresión son del doble comparados con los encontrados en población general (Paukert, LeMaire, y Cully, 2009). 
Diferentes cambios en los estados anímicos (e.g. ansiedad, depresión, ira) se identifican en diversas condiciones pre y post operatorias (Ozdemir et al., 2015) y después de eventos cardiacos (e.g. infartos) (Bennett et al., 2002). En términos de antecedentes sociodemográficos, no se reportan diferencias significativas en síntomas depresivos en función del estado civil y/o del apoyo social (Paukert, LeMaire, y Cully, 2009). Mediciones con la escala POMS revelan que, comparadas con los varones, son las mujeres con ECV quienes presentan mayores puntuaciones de depresión e ira (Ozdemir et al., 2015). Evangelista et al. (2008) señala la no existencia de diferencias por sexo y edad con respecto a los niveles de fatiga emocional reportados por un grupo de pacientes con insuficiencia cardiaca. Fennessy et al. (2010) considerando una muestra de pacientes de infarto agudo del miocardio, refiere que son las mujeres quienes reportan una mayor fatiga afectiva en contraste con los varones.

De la revisión anterior se desprende que existe una amplia tradición para documentar el perfil afectivo en pacientes con cáncer, dejando de lado la investigación sobre el perfil afectivo y su relación con antecedentes sociodemográficos y clínicos en otro tipo de ECnT cuyos factores de riesgo, costos sociales y económicos son similares. Asimismo, la evaluación del perfil anímico tiende a enfocarse más en estados de ánimo negativos (e.g. ansiedad, depresión y fatiga), dejando de lado la caracterización de estados de ánimo positivos (Lane, 2014).

En virtud de lo anterior, el presente estudio tiene como propósito comparar el impacto psicológico de las enfermedades crónicas: cáncer, IR, diabetes y ECV en los estados de ánimo de diferentes muestras de pacientes adultos mexicanos, tomando en cuenta también su relación con variables clínicas y sociodemográficas. Específicamente, se examina el perfil anímico de los pacientes con dichas ECnT considerando las dimensiones clínicamente relevantes de estados de ánimo: tensión, depresión, ira, vigor, fatiga y confusión. Asimismo, considerando que un IPE ofrece información práctica y clínica, se describen los IPE basados en los aspectos clínicos de la enfermedad y los antecedentes sociodemográficos de las submuestras en cuestión. 


\section{Método}

\section{Participantes}

El total de la muestra consistió en 386 participantes distribuidos en las cuatro categorías de enfermedades crónicas: pacientes con cáncer $(n=96)$, IR $(n=107)$, diabetes $(n=91)$ y con ECV $(n=92)$. Todos ellos fueron admitidos y diagnosticados clínicamente en la Unidad Médica de Alta Especialidad No. 1 (UMAE) del Instituto Mexicano del Seguro Social (IMSS) del municipio de León, estado mexicano de Guanajuato. Los criterios de inclusión para los subgrupos de pacientes fueron: estar recibiendo atención médica en la UMAE, ser mayor de 17 años, ser capaces de leer y escribir, tener un diagnóstico previo de algunas de las ECnT. Por otra parte, se excluyeron a aquellos pacientes con déficits cognitivos perceptibles, desordenes de lenguaje $\mathrm{u}$ otras alteraciones en su estado mental que pudieran repercutir en las evaluaciones del estado afectivo y la emisión del consentimiento informado.

\section{Consideraciones éticas}

El presente estudio formó parte de un protocolo de investigación previamente aprobado por el Comité de Ética en Investigación del IMSS, Hospital de Especialidades No. 1, Bajío, León, Guanajuato (R-2020-1001-010). Todos los participantes otorgaron su consentimiento informado por escrito para participar de manera anónima y voluntaria y sin compensación económica alguna.

\section{Instrumentos}

El registro de datos sociodemográficos consistió en la recopilación de los antecedentes: sexo, edad, escolaridad, estado civil, ocupación y religión. En las características clínicas se incluyó información relevante relacionada con la naturaleza de la enfermedad, su curso y/o evolución, así como tipo de tratamiento según el caso. Para los pacientes con cáncer se consideró la tipología, presencia de dolor (agudo o crónico), etapa de cáncer y tratamiento actual. En los pacientes con IR se evalúo el tipo 
de insuficiencia (aguda o crónica) y la fase de tratamiento. En pacientes diabéticos se incluyó la valoración del tipo de diabetes (I o II) y el auto reporte de las últimas dos semanas de los valores de glucosa en sangre. En el caso de los pacientes con ECV se consideró el tipo de procedimiento realizado (cateterismo cardiaco, angioplastia, colocación de marcapaso, valvulopatía mitral o trombectomía) así como la presencia de comorbilidad. La evaluación de los estados de ánimo consistió en la aplicación de la versión al castellano de la escala POMS (Andrade, Arce, y Seoane, 2002) la cual consta de las dimensiones de Tensión (8 ítems), Depresión (14 ítems), Cólera (12 ítems), Vigor (8 ítems), Fatiga (7 ítems), Confusión (7 ítems) y Amistad (7 ítems) y un formato de respuesta de 5 puntos $(1=$ para nada, hasta 5 = extremadamente $)$. Un índice de malestar emocional general o IPE se obtiene mediante la fórmula depresión + tensión + cólera + fatiga + confusión - vigor.

\section{Procedimiento}

La aplicación de los instrumentos se realizó en forma individual mediante un protocolo estándar de instrucciones por cada aplicación considerando las instalaciones de la UMAE. Un entrevistador capacitado obtuvo el consentimiento informado de los participantes y explicó la finalidad de la investigación y las instrucciones. En la obtención del perfil se siguió la instrucción habitual "cómo se ha sentido durante la última semana, incluyendo el día de hoy” (McNair et al., 1971). La aplicación tuvo una duración aproximada de 15 minutos.

\section{Análisis de datos}

Se empleo estadística descriptiva para la caracterización y respectiva representación tabular de las variables sociodemográficas, clínicas y del perfil anímico. Análisis de consistencia interna de las dimensiones de la escala POMS se llevaron a cabo usando el índice alfa de Cronbach. La normalidad de la distribución de los datos de la muestra fue analizada con la prueba de Kolmogorov-Smirnov y la homocedasticidad con el tets de Levene. Debido a que la distribución de los puntajes no tuvo 
una distribución normal, análisis subsecuentes de comparaciones entre los grupos de variables se llevaron a cabo mediante análisis no paramétricos para grupos independientes de comparación de dos o más grupos (test de Mann-Whitney y Kruskal-Wallis respectivamente). Asimismo, análisis no paramétricos de correlación Rho Spearman fueron llevados a cabo para conocer la relación entre la edad y los índices de perturbación emocional de los grupos de pacientes.

Las diferencias en perfil anímico de los grupos diagnósticos con respecto a las dimensiones de la escala POMS (tensión, depresión, cólera, vigor, fatiga, confusión, amistad) y el indice total de perturbación emocional fueron analizadas mediante la prueba de Kruskal-Wallis. En todos estos casos, las pruebas de seguimiento post hoc de los contrastes fueron realizados con la prueba de Mann-Whitney utilizando el método de corrección de Bonferroni para contrarrestar los efectos de las comparaciones múltiples (Field, 2013), reportándose también la magnitud de las diferencias (tamaño del efecto TE) a través del coeficiente $r$ (ver Rosenthal y DiMatteo, 2001).

\section{Resultados}

Los valores alfa de Cronbach para los factores de la escala POMS que se obtuvieron en la presente investigación mostraron valores de $\propto=.89$ para Tensión; $\propto=.96$ para Depresión; $\propto=.96$ para Cólera; $\propto=.81$ para Vigor; $\propto=.88$ para Fatiga; $\propto=.90$ para Confusión y $\propto=.78$ para Amistad.

\section{Perfil sociodemográfico de los pacientes con ECnT}

La tabla 1 da cuenta del registro de los datos sociodemográficos respecto a las particularidades de los cuatro subgrupos. En estos se observan un total de $N=386$ participantes jóvenes y adultos (rango de edad: 17-82 ańos; edad media 55.44 ańos; $D E=12.78$ ) que en su conjunto aceptaron participar. En los pacientes cáncer e IR un 62.5\% y $50.5 \%$ de los casos estuvo compuesto de mujeres, mientras que en 
los grupos de diabetes y ECV existió una mayor prevalencia de varones (64.8\% y $63 \%$ respectivamente).

Por otra parte, respecto a las demás características sociodemográficas, se observa que la muestra en general reportó una escolaridad tendiente a ubicarse en rangos de escolaridad menores a 12 años, una mayor prevalencia de participantes casados $(59.6 \%)$ y ocupaciones preponderantes de empleado (43.3\%), ama de casa (19.2\%) y comerciantes (14.5\%), siendo todos en su mayoría de religión católica (ver Tabla 2).

\section{Tabla 1}

Perfil sociodemográfico de los grupos diagnósticos con ECnT

\begin{tabular}{|c|c|c|c|c|}
\hline \multirow[b]{2}{*}{ Variables sociodemográficas } & \multicolumn{4}{|c|}{ Grupo diagnóstico } \\
\hline & CA & IR & D & ECV \\
\hline Pacientes $f(\%)$ & $96(24.9)$ & $107(27.7)$ & $91(23.6)$ & $92(23.8)$ \\
\hline \multicolumn{5}{|l|}{ Edad } \\
\hline Rango & $17-76$ & $18-81$ & $44-82$ & $50-74$ \\
\hline Promedio $(D E)$ & $54.1(12.3)$ & $46.2(15.3)$ & $61.6(6.7)$ & $61.5(5.4)$ \\
\hline \multicolumn{5}{|l|}{ Sexo $f(\%)$} \\
\hline Femenino & $60(62.5)$ & $54(50.5)$ & $32(35.2)$ & $34(37)$ \\
\hline Masculino & $36(37.5)$ & $53(49.5)$ & $59(64.8)$ & $58(63)$ \\
\hline \multicolumn{5}{|l|}{ Escolaridad $f(\%)$} \\
\hline Ninguna & $13(13.5)$ & $5(4.7)$ & $15(16.5)$ & $5(5.4)$ \\
\hline Primaria & $31(32.3)$ & $38(35.5)$ & $41(45.1)$ & $35(38)$ \\
\hline Secundaria & $33(34.4)$ & $37(34.6)$ & $29(31.9)$ & $40(43.5)$ \\
\hline Medio superior & $9(9.4)$ & $21(19.6)$ & $6(6.6)$ & $10(10.9)$ \\
\hline Licenciatura & $8(8.3)$ & $5(4.7)$ & $0(0)$ & $2(2.2)$ \\
\hline No contestó & $2(2.1)$ & $1(.9)$ & $0(0)$ & $0(0)$ \\
\hline \multicolumn{5}{|l|}{ Estado civil $f(\%)$} \\
\hline Soltero (a) & $15(15.6)$ & $24(22.4)$ & $10(11)$ & $2(2.2)$ \\
\hline Casado (a) & $56(58.3)$ & $69(64.1)$ & $55(60.4)$ & $50(54.3)$ \\
\hline Unión libre & $0(0)$ & $1(.9)$ & $0(0)$ & $4(4.3)$ \\
\hline
\end{tabular}




\begin{tabular}{|c|c|c|c|c|}
\hline \multirow[b]{2}{*}{ Variables sociodemográficas } & \multicolumn{4}{|c|}{ Grupo diagnóstico } \\
\hline & CA & IR & $\mathrm{D}$ & $\mathrm{ECV}$ \\
\hline Divorciado (a) & $11(11.5)$ & $6(5.6)$ & $12(13.2)$ & $14(15.2)$ \\
\hline Viudo (a) & $13(13.5)$ & $7(6.5)$ & $14(15.4)$ & $22(23.9)$ \\
\hline No contestó & $1(1.0)$ & $0(0)$ & $0(0)$ & $0(0)$ \\
\hline \multicolumn{5}{|l|}{ Ocupación $f(\%)$} \\
\hline Estudiante & $1(1)$ & $2(1.9)$ & $0(0)$ & $0(0)$ \\
\hline Ama de casa & $35(36.5)$ & $27(25.2)$ & $8(8.8)$ & $4(4.3)$ \\
\hline Empleado (a) & $24(25)$ & $42(39.3)$ & $60(65.9)$ & $41(44.6)$ \\
\hline Comerciante & $7(7.3)$ & $15(14)$ & $6(6.6)$ & $28(30.4)$ \\
\hline Pensionado (a) & $7(7.3)$ & $2(1.9)$ & $0(0)$ & $3(3.3)$ \\
\hline Jubilado (a) & $5(5.2)$ & $0(0)$ & $1(1.1)$ & $7(7.6)$ \\
\hline Obrero (a) & $9(9.4)$ & $13(12.1)$ & $16(17.6)$ & $9(9.8)$ \\
\hline Profesionista & $1(1)$ & $0(0)$ & $0(0)$ & $0(0)$ \\
\hline No contestó & $7(7.3)$ & $4(3.7)$ & $0(0)$ & $0(0)$ \\
\hline \multicolumn{5}{|l|}{ Religión $f(\%)$} \\
\hline Ninguna & $2(2.1)$ & $3(2.8)$ & $4(4.4)$ & $4(4.3)$ \\
\hline Católica & 87 (90.6) & $95(88.8)$ & $86(94.5)$ & 87 (94.6) \\
\hline Cristiana & $2(2.1)$ & $2(1.9)$ & $1(1.1)$ & $1(1.1)$ \\
\hline No contestó & $5(5.2)$ & $5(4.7)$ & $0(0)$ & $0(0)$ \\
\hline
\end{tabular}

Nota: CA = Cáncer, IR = Insuficiencia renal, D = Diabetes, ECV = Enfermedad cardiovascular

\section{Perfil clínico de los pacientes con ECnT}

La tabla 2 contiene los parámetros clínicos de relevancia de los subgrupos de pacientes. En la submuestra de pacientes cáncer se identifica la prevalencia de cinco tipologías de cáncer (mama, gástrico, colón, pulmón y próstata) que en su conjunto abarcan más del $86 \%$ de los casos. La mayoría de los casos reportó tener episodios de dolor agudo $(42.7 \%)$ y estar tanto en etapa intermedia de la enfermedad (15\%) como en etapa final ( $V$ con $22.7 \%)$. Se observa que la mayoría de ellos 
(52\%) no identificaba el estadio en el que se ubicaba su enfermedad. Para estos pacientes, la mayor modalidad recibida de tratamiento era la quimioterapia (74\%). En cuanto a los pacientes con IR casi todos eran pacientes con insuficiencia del tipo crónica (96\%) y en tratamiento de diálisis (48.6). En los pacientes con ECV se identifica que varios de ellos recibieron tratamientos médicos asociados al cateterismo cardiaco y marcapasos (31.5\% respectivamente). De estos, un 63\% había tenido algún tipo de procedimiento quirúrgico del corazón. Un $66.6 \%$ cursaba con un cuadro de concurrencia con respecto a otras enfermedades, entre ellas hipertensión (46.7\%) y diabetes (19.6\%). Finalmente, la muestra de pacientes diabetes se conformó en mayor medida de pacientes con diabetes tipo I (81.3\%) con valores promedio autoreportados de glucosa (últimas dos semanas) de $261 \mathrm{mg} / \mathrm{dl}$.

\section{Tabla 2}

Perfil clinico de los grupos de pacientes con EcnT

\begin{tabular}{|c|c|c|c|c|c|}
\hline Grupo DX & Perfil clínico & $f(\%)$ & $\begin{array}{c}\text { Grupo } \\
\text { DX }\end{array}$ & Perfil clínico & $f(\%)$ \\
\hline \multirow[t]{10}{*}{$\mathrm{CA}$} & Tipo de cáncer & & IR & Tipo de insuficiencia & \\
\hline & Próstata & $15(15.6)$ & & Aguda & $10(9.3)$ \\
\hline & Mama & $17(17.7)$ & & Crónica & $96(89.7)$ \\
\hline & Gástrico & $17(17.7)$ & & Tipo de tratamiento & \\
\hline & Pulmón & $16(16.7)$ & & Predialisis & $10(9.3)$ \\
\hline & Colón & $17(17.7)$ & & Diálisis & $52(48.6)$ \\
\hline & Cérvico uterino & $11(11.5)$ & & Hemodiálisis & $28(26.2)$ \\
\hline & Laringe & $1(1)$ & & Trasplante renal & $17(15.8)$ \\
\hline & Linfoma de hodkin & $2(2.1)$ & & & \\
\hline & Presencia de dolor & & & Tipo de intervención & \\
\hline
\end{tabular}




\begin{tabular}{|c|c|c|c|c|c|}
\hline Grupo DX & Perfil clínico & $f(\%)$ & $\begin{array}{c}\text { Grupo } \\
\text { DX }\end{array}$ & Perfil clínico & $f(\%)$ \\
\hline & Agudo & $41(42.7)$ & ECV & Cateterismo cardiaco & $29(31.5)$ \\
\hline & Crónico & $25(26)$ & & Angioplastia & $23(25)$ \\
\hline & Sin dato & $30(31.3)$ & & Implante de marcapasos & $29(31.5)$ \\
\hline & Etapa de cáncer & & & Valvulopatia mitral & $5(5.4)$ \\
\hline & $\mathrm{I}$ & $7(8)$ & & Trombectomia quirúrgica & $6(6.5)$ \\
\hline & II & $8(9.1)$ & & & \\
\hline & III & $14(15)$ & & Presencia de comorbilidad & \\
\hline & IV & $7(8)$ & & $\mathrm{Si}$ & $61(66.7)$ \\
\hline & $\mathrm{V}$ & $20(22.7)$ & & No & $31(33.7)$ \\
\hline & No sabe & $52(36.4)$ & & Clasificación & \\
\hline & & & $\mathrm{D}$ & Tipo I & $74(81.3)$ \\
\hline & Tipo de tratamiento & & & Tipo II & $17(18.7)$ \\
\hline & Quimioterapia & $71(74)$ & & $\begin{array}{l}\text { Glucos sangre Promedio } \\
(D E)\end{array}$ & $261(43.12)$ \\
\hline & Radiación & $2(2.1)$ & & & \\
\hline & Mixta & $21(21.9)$ & & & \\
\hline & Otro & $1(1)$ & & & \\
\hline
\end{tabular}

Nota $:$ CA = Cáncer, IR = Insuficiencia renal, D = Diabetes, ECV = Enfermedad cardiovascular

\section{Análisis de la relación entre las variables sociodemográficas con respecto al IPE en función de los grupos diagnósticos}

Los análisis de correlación revelan la existencia de asociaciones significativas entre la edad y el IPE en los pacientes con IR y diabetes. En específico, se observa que en los pacientes con IR una mayor edad se relaciona con mayor perturbación emocional $\left(r_{s}=.30, p<.00\right)$, mientras que en los pacientes diabéticos a mayor edad menor perturbación emocional $\left(r_{\mathrm{s}}=-.23, p<.00\right)$. No se reportan correlaciones con el resto de los grupos diagnósticos. Con respecto al sexo, contrastes con la prueba de Mann-Whitney para muestra de grupos independientes denotan que el 
IPE en varones no difiere significativamente del de las mujeres en todos los subgrupos clínicos a saber: cáncer $(U=957.50, z=-.27, p=.35)$, insuficiencia renal $(U=1372, z=-.36, p=.71)$, diabetes $(U=770.50$, $z=-1.44, p=.14)$ y $\operatorname{ECV}(U=920, z=-.53, p=.59)$. En la tabla 3 se observa que, en los pacientes con cáncer, aquellos con una mayor escolaridad tienden a presentar menores puntajes de malestar emocional comparados con quienes tienen menos años de instrucción $(p<.05)$. Contrariamente, es en los subgrupos de los pacientes con IR y diabetes en donde se observan diferencias estadísticamente significativas entre aquellos con mayores niveles de escolaridad con respecto a quienes no la tienen $(p<.05)$, siendo los subgrupos con ausencia de escolaridad quienes presentan menores puntajes de perturbación emocional. No se documentan diferencias significativas entre los subgrupos de pacientes con ECV con respecto a los niveles de escolaridad y el IPE. En cuanto a las variables asociadas al estado civil no se identifican diferencias estadísticamente significativas (datos no mostrados en la Tabla 3) con respecto al IPE para los cuatro grupos de ECnT $(p>.05)$. Con relación a la ocupación, en general aquellos pacientes que cuentan con bajos niveles de ocupación laboral presentan mayores IPE. En pacientes con ECV, después de un bajo nivel de ocupación laboral, son los pacientes jubilados quienes presentan mayores IPE al compararse con aquellos con ocupación de comerciante y empleado $(p>.00)$. 
Perfil anímico en pacientes con enfermedades crónicas / Martínez-Soto y Ramos-Frausto

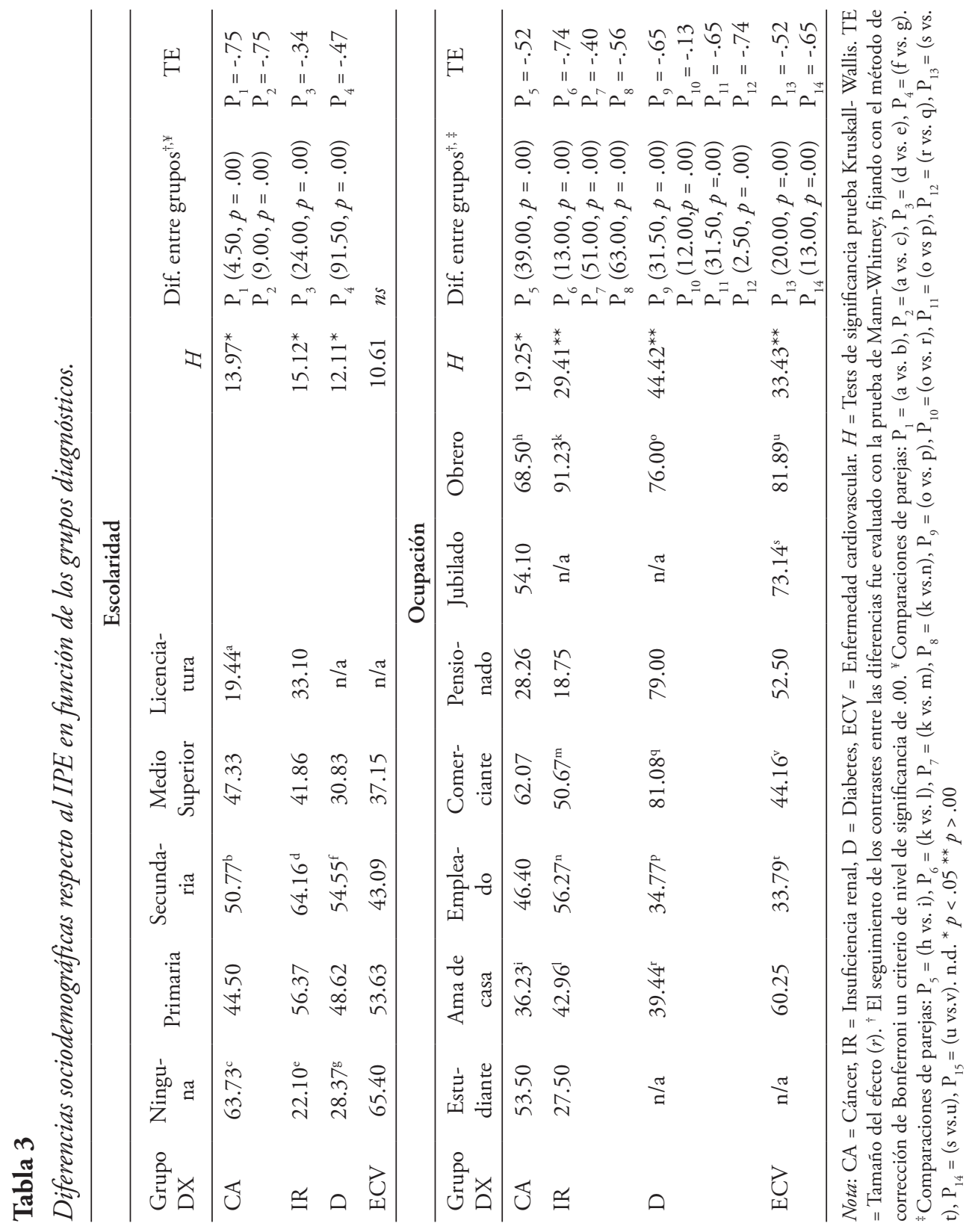


Comenzando con el grupo de pacientes cáncer, contrastes con la prueba de Mann-Whitney para muestras de grupos independientes denotan que no existen diferencias en cuanto a los niveles de perturbación emocional de aquellos pacientes que experimentan dolor agudo $(M d n=43.33)$ vs. crónico $(M d n=32.14-M d n)(U=478.50, p=.65)$. Asimismo, la tabla 4 muestra que no existen diferencias estadísticamente significativas entre los diferentes tipos de cáncer con respecto al IPE $(p>.05)$. Respecto a las etapas de cáncer, el grupo que no cuenta con conocimientos sobre el grado de avance de la enfermedad presenta menores puntuaciones totales de perturbación emocional al compararse con el grupo que reporta estar en estadios más avanzados (etapa V; $p<.00)$. De la misma forma, es el subgrupo con estadios más avanzados de la enfermedad (etapa V) quien presenta un mayor IPE al compararse con aquellos subgrupos de estadios menos avanzados $(p<.05)$. En cuanto a los pacientes con IR, se observan contrastes significativos entre los pacientes que se encuentran en tratamiento de diálisis vs. aquellos en situación de trasplante renal $(p<.05)$, siendo estos quienes presentan menores IPE, incluso con puntajes relativamente similares a los de una situación inicial de prediálisis. Finalmente, no se observan diferencias significativas entre los subgrupos de los pacientes ECV en los IPE con respecto al tipo de intervención (Tabla 4).

\section{Diferencias en el perfil animico respecto a los grupos diagnósticos de enfermedades crónicas}

La tabla 5 muestra un análisis entre grupos de las diferencias en el perfil anímico de los grupos diagnósticos considerando, además del IPE, las siete dimensiones de la escala POMS. Una ilustración del perfil anímico intragrupos se muestra en la figura 1. En general, la prueba de Kruskall-Wallis revela la existencia diferencias estadísticamente entre los grupos respecto a las dimensiones de Tensión $([\mathrm{H3}]=40.85$, $p<.00)$, Depresión $([H 3]=54.21, p<.00)$, Cólera $([H 3]=42.78$, $p<.00)$, Vigor $([H 3]=21.81, p<.00)$, Fatiga $([H 3]=39.32, p<.00)$, Confusión $([H 3]=43.56, p<.00)$; Amistad $([H 3]=15.89, p<.00)$ y el IPE $([H 3]=43.75, p<.00)$. Análisis post hoc de seguimiento con 
la prueba de Mann-Whitney para muestras de grupos independientes, refieren que los grupos de cáncer e IR no presentan diferencias estadísticamente significativas $(p>.05)$ en cuanto a sus niveles moderados de puntajes de todas las dimensiones de la escala POMS (Tensión, Depresión, Cólera, Vigor, Fatiga, Confusión, Amistad) y el IPE. Como se observa en los análisis ulteriores, estos puntajes tienden a ser menores al compararse con los autoreportados por los grupos de diabetes y ECV. Asimismo, entre estos dos grupos, son los pacientes con ECV quienes presentan mayores puntajes en las todas las dimensiones del perfil anímico y el IPE al compararse con los pacientes diabetes (ver Tabla 5). Respecto a la primera dimensión positiva de Vigor, las diferencias estadísticamente significativas en los grupos diagnósticos denotan la existencia de diferencias entre los pacientes con cáncer, IR y diabetes con respecto al grupo de ECV, siendo este último el que mayores puntuaciones reporta. En lo tocante a la siguiente dimensión positiva de la escala POMS, amistad, se observa la existencia de diferencias entre el grupo de diabetes vs. de ECV, siendo este último quien mayor puntaje obtiene para dicha dimensión (ver Tabla 5).

PACIENTES CON CÁNCER

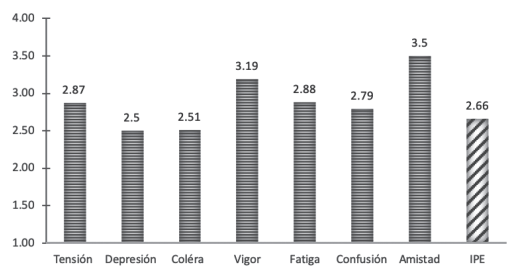

PACIENTES CON INSUFICIENCIA RENAL

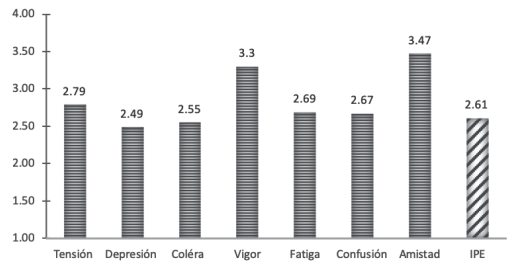

PACIENTES CON DIABETES

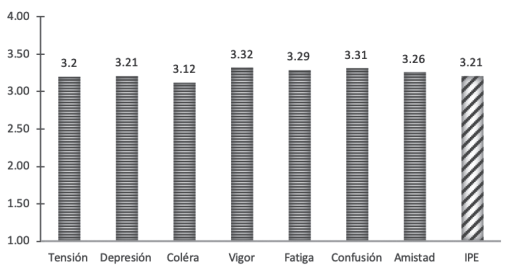

PACIENTES CON ENFERMEDAD CARDIOVASCULAR

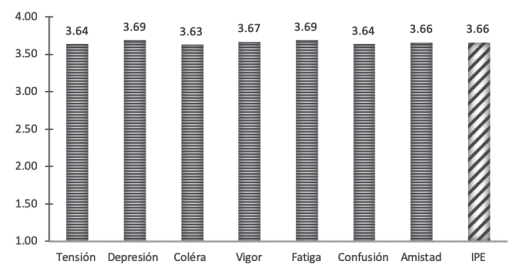

Figura 1. Perfil anímico de los grupos diagnóstico de los grupos con ECnT 


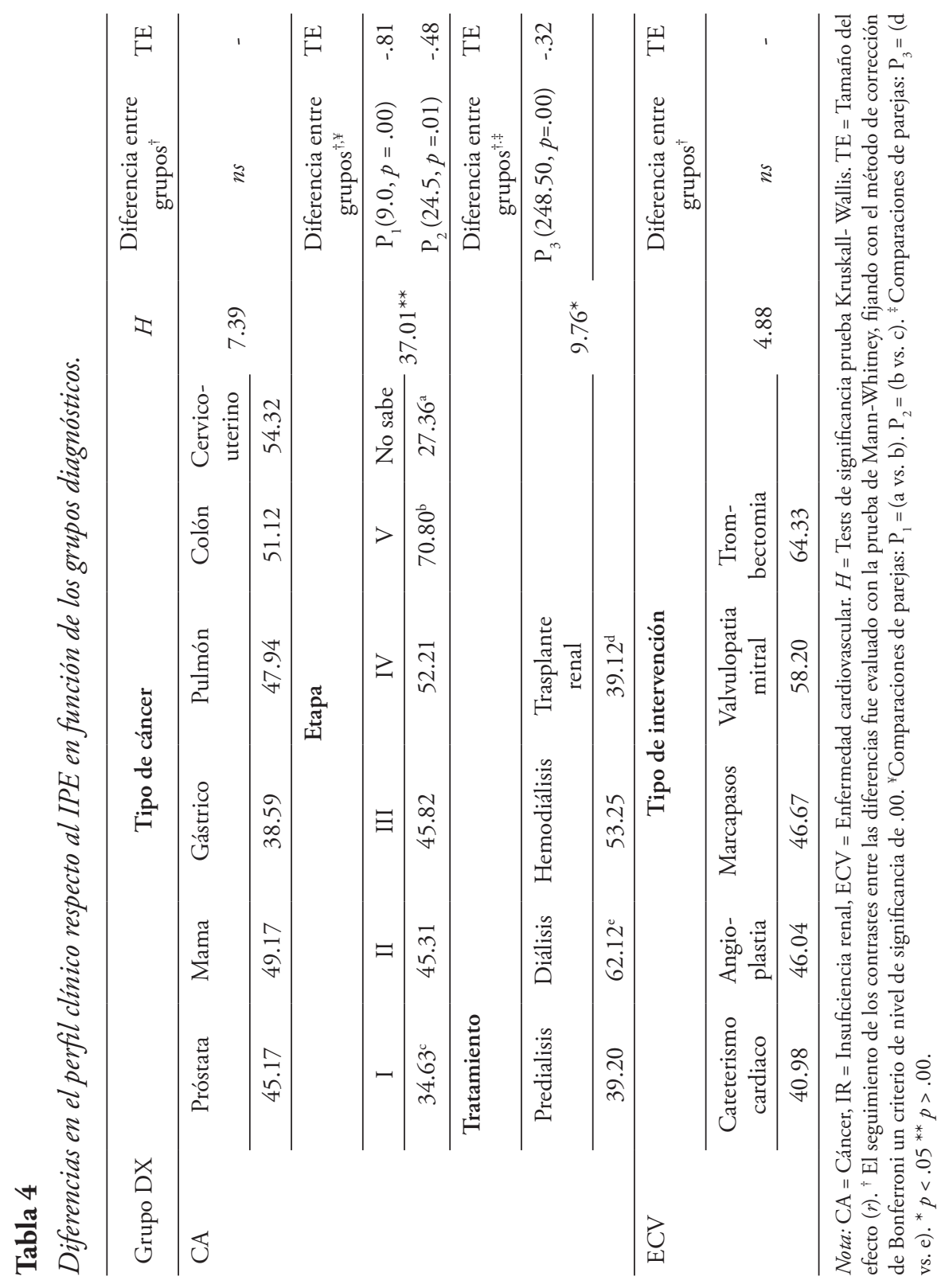




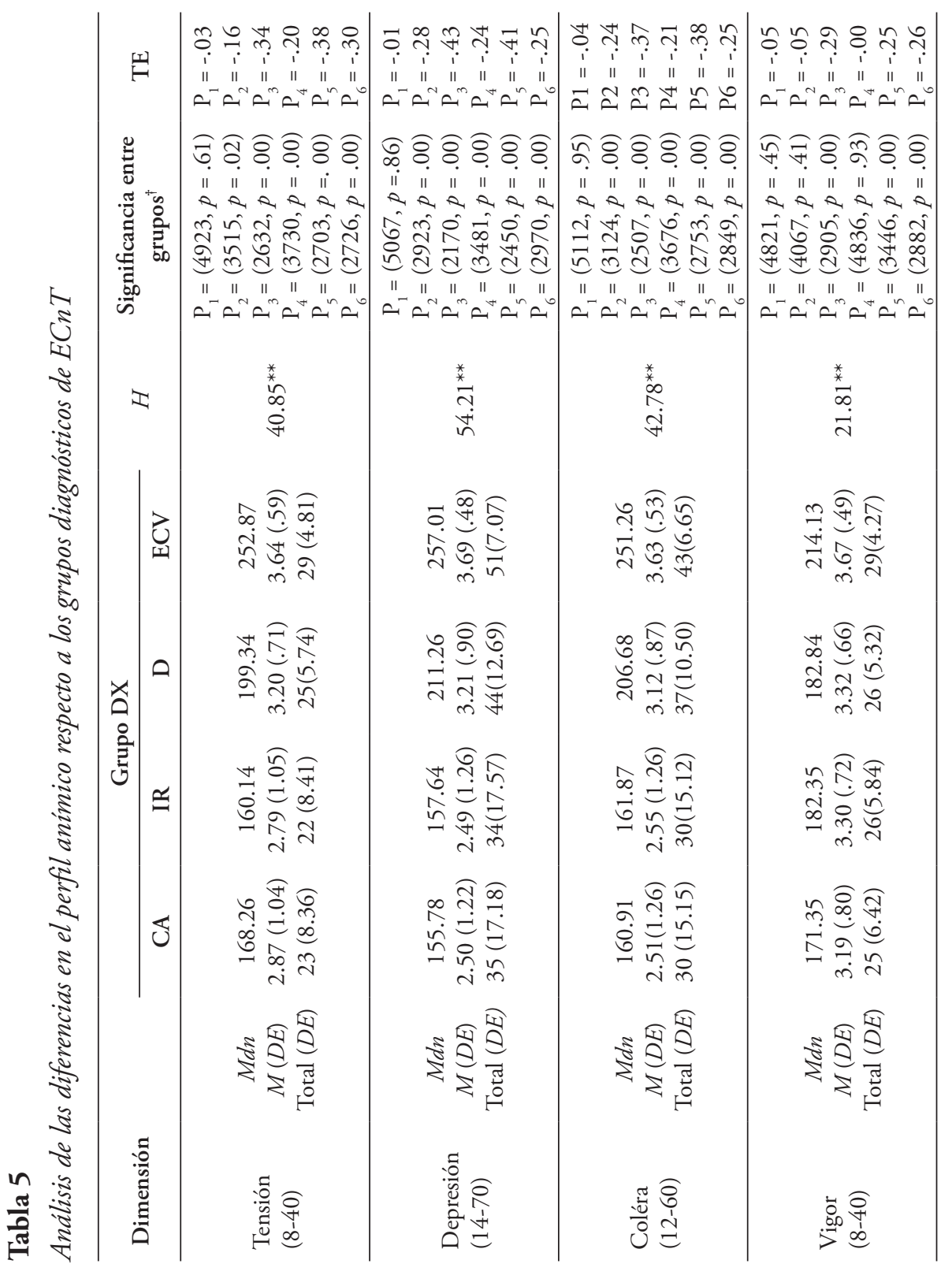




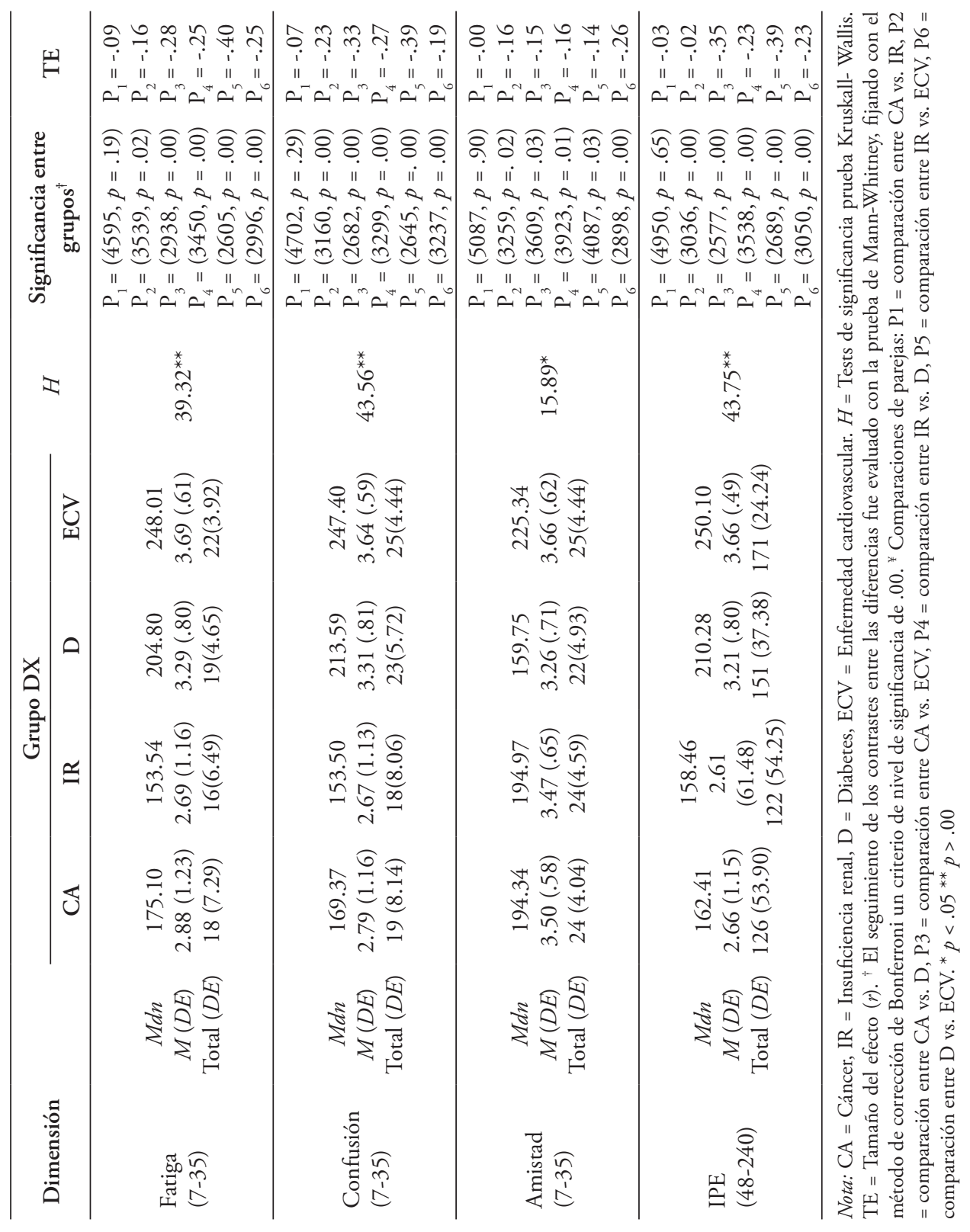




\section{Discusión}

El objetivo del presente estudio consistió en documentar el perfil anímico de cuatro ECnT y su asociación con variables sociodemográficas y clínicas. Se discute a continuación los hallazgos cuantitativos y cualitativos que responden a el objetivo planteado.

Comenzando con los antecedentes sociodemográficos, los resultados revelan que no existen diferencias en el IPE con respecto al estado civil y sexo de las cuatro submuestras de pacientes. Si bien en el presente estudio, el estatus marital no ejerce influencia significativa en el perfil anímico de los pacientes, debe de considerarse la influencia de otras variables afines no consideradas en esta investigación, entre ellas la edad en la que se contrajo el matrimonio (McFarland, Hayward, y Brown, 2013) y los años de relación (Dupre, Beck, y Meadows, 2009). Con relación al sexo, los resultados contrastan con la literatura empírica previa de la escala POMS en donde se ha documentado que, por lo general, son las mujeres con ECnT quienes, en contraste con los varones, tienden a presentar mayores perturbaciones emocionales (Annunziata et al., 2013; Ozdemir et al., 2015).

La literatura refiere que un mayor nivel educativo propicia un mejor entendimiento y atención a la enfermedad, reduciendo el estrés psicológico (Rometsch et al., 2018). Los hallazgos indican que esta condición podría asemejarse con los pacientes con cáncer, quienes reportan que, a mayor escolaridad, menores IPE. Contrariamente, son los pacientes con IR y diabetes, quienes refieren que, a menor escolaridad, menor es la existencia de malestar emocional, lo cual contrasta con lo encontrado en investigaciones previas (Keskin y Engin, 2011; Paez et al., 2009). Al respecto, la teoría de cambio de respuesta (Sprangers y Schwartz, 1999) refiere que las personas pueden adaptarse a su condición mediante la modificación de valores internos que les permitan mantener una adecuada salud mental a pesar de sus impedimentos físicos o funcionales (Ilie et al., 2019). En dicha adaptación el nivel educativo puede o no desempeñar un papel relevante (Kao et al., 2020). 
En cuanto a la edad, se observa que a mayor edad en los pacientes con IR existe una mayor afectación emocional, y contrariamente en los pacientes con diabetes a más edad menos perturbación emocional. Diversos aspectos psicológicos relacionados con las estrategias de afrontamiento, regulación emocional y mayor resiliencia en personas diabéticas de mayor edad, pueden explicar en parte dichos resultados (Martins-Klein et al., 2019).

Por otra parte, los resultados del presente estudio denotan que un bajo nivel socioeconómico se asocia con mayor IPE en todos los grupos, dando cuenta de los efectos adversos de un bajo nivel socioeconómico a una pobre adaptación a la enfermedad (Helgeson y Zajdel, 2017). Continuando con algunos antecedentes clínicos de las ECnT y sus influencias en el perfil afectivo de los pacientes, se observa que contrario a lo documentado en la literatura, no existen diferencias en cuanto a los tipos de cáncer y el IPE (Allart-Vorelli et al., 2015; Nikbakhsh et al.,2014). Estos hallazgos podrían deberse a que se cuenta con una muestra heterogénea de diagnósticos y cantidad limitada de casos. Asimismo, las evidencias de la investigación denotan que en general, la presencia de dolor (agudo o crónico) no tiene influencia significativa alguna en el IPE de los pacientes con cáncer, lo que difiere de estudios previos (Lin, Lai, y Ward, 2003; Wang et al., 2007).

En el presente estudio se ha encontrado también que el hecho de no contar con información sobre el estadio de la enfermedad y de la misma forma, estar en estadios más avanzados de cáncer se asocia con mayor malestar emocional, lo que coincide con una mayor prevalencia de trastornos emocionales post diagnóstico y su asociación con etapas más tardías de cáncer (Vodermaier et al., 2011). En cuanto a los pacientes con disfunción renal, se observa que el impacto psicológico negativo de la enfermedad se reduce significativamente en aquellos pacientes con trasplante renal, lo cual podría estar asociado a una mejora en su condición médica y de salud (Tamura et al., 2018).

En esta investigación se observa que, en los pacientes con diabetes, las variaciones de glucosa no tienen influencia alguna en el IPE, lo que contrasta con la literatura antecedente, en donde se ha documentado 
que fluctuaciones en los niveles de glucosa pueden tener efectos negativos en los estados de ánimo (Arab et al., 2018; Fritschi y Quinn, 2010). A manera de explicación, podría considerarse que los efectos de la glucosa en los estados de ánimo pueden ser moderados por diferencias individuales del control glucoregulatorio (Mantantzis et al., 2019). Además, en lo tocante a los pacientes con ECV, no se observan diferencias en el IPE con respecto a el tipo de intervención médica proporcionada. Parte de la explicación para estos hallazgos, tendría que ver con el hecho de que no se cuenta con información del perfil anímico previo a el tipo de intervención planteada, limitando el abordaje de inferencias causales (Roberts y Ilardi, 2003).

En alusión a las diferencias en el perfil anímico respecto a los grupos diagnósticos de enfermedades crónicas, en el presente estudio, se ha encontrado que el perfil anímico de las ECnT parece ser dependiente del tipo de enfermedad, con unas mayores afectaciones emocionales en pacientes con ECV y diabéticos. Lo anterior contrasta con investigaciones previas donde, por una parte, los grupos de pacientes diabéticos y de ECV tienden a presentar menores malestares emocionales relacionados con su condición y, por otra, los pacientes con cáncer refieren mayores problemas emocionales al compararse con aquellos pacientes con trastornos cardiovasculares (McCorkle y Quint-Benoliel, 1983; Penninx et al., 1996).

La presente investigación se caracteriza por contar con un subgrupo mayoritario de pacientes con diabetes tipo I (81.3\%). Algunos estudios refieren que comparados con los pacientes con diabetes tipo II, las personas con diabetes tipo I tienden a presentar mayores complicaciones emocionales (e.g. menos vitalidad, motivación reducida, fatiga crónica) (Jensen et al., 2018). Sobresale el hecho también de que son los pacientes con ECV quienes reportan una mayor afectación anímica si se compara con pacientes diabéticos y el resto de los subgrupos.

Dos posibles factores podrían estar relacionados con una mayor afectación emocional en esta submuestra, entre ellos la alta presencia de comorbilidad (hipertensión y diabetes; Moe, 2016) y los factores de riesgo psicosocial emocional catalizadores del desarrollo pre y post de 
la enfermedad (depresión, ansiedad y hostilidad; Schneiderman et al., 2001). Estos aspectos aunados a el contexto socioeconómico podrían en parte explicar una mayor prevalencia de problemas emocionales. Se encuentra que los pacientes con cáncer e IR, jóvenes y adultos, varones y mujeres, con diferentes antecedentes clínicos presentan un perfil anímico similar. Es decir, niveles moderados de estados de ánimo negativos (tensión, depresión, colera, fatiga, confusión), positivos (vigor y amistad) e IPE semejantes.

Los hallazgos encontrados con respecto a los pacientes con cáncer difieren de lo reportado en la literatura, en donde el perfil afectivo de este tipo de pacientes (evaluado a través de la escala POMS) se caracteriza por tener bajas puntuaciones en la escala de ira y vigor y altas puntuaciones de ansiedad, confusión, depresión y fatiga (Barinková, y Mesároová, 2013; Koizumi et al., 2018; Groarke et al., 2018; Jiang et al., 2018; Lin et al., 2003; Sasai y Onishi, 2017). Por otra parte, el estudio de los aspectos anímicos de los pacientes con IR suele centrarse en abordar a la fatiga y depresión como dos problemas psicológicos recurrentes para dicha población.

La captura de los resultados y su contraste con los otros grupos diagnósticos complementa empíricamente la información relacionada con el perfil anímico para este tipo de pacientes. Para estos y otros hallazgos afines debe de considerarse los aspectos comunes y no comunes a la enfermedad. Entre los primeros se destaca la existencia de preocupaciones propias de la enfermedad crónica (e.g. temor e incertidumbre, limitaciones físicas y preocupación por el dolor) (Stanton, Revenson, y Tennen, 2007). En cuanto a los segundos, se debe tomar en cuenta que las respuestas emocionales ante la enfermedad crónica son dinámicas y pueden depender de la severidad de la enfermedad, pronóstico, tiempo de deterioro de la salud, así como de los periodos sintomáticos y asintomáticos.

Respecto a la caracterización del afecto positivo, si bien los pacientes con ECV tienden a reportar mayores niveles de malestar emocional a lo largo de las dimensiones de la escala POMS, también obtienen mayores puntuaciones en las dimensiones positivas de Vigor 
y Amistad. A manera de antecedente, debe referirse que un 63\% de los pacientes de la submuestra tienen un seguimiento médico post quirúrgico, representando una muestra en situación de restablecimiento de la enfermedad. Si bien algunas investigaciones documentan la existencia de estrés y estados anímicos negativos pre y post recuperación quirúrgica (e.g. depresión y ansiedad; Andrew et al., 2000; Horne et al., 2013; Neupane, Arora, y Rudolph, 2017), otras investigaciones señalan la existencia de efectos psicológicos positivos post intervención quirúrgica (Kiecolt-Glaser et al., 1998).

Stanton et al. (2007) examina los procesos inherentes que son resultado de un ajuste psicológico ante la enfermedad, entre ellos aquellos relacionados con la presencia del afecto positivo. Una predisposición relacionada con dicho ajuste es el optimismo (variable asociada a la sensación de vigor; Brydon et al., 2009) el cual independientemente de estados psicológicos previos como la ansiedad y depresión, se asociado con una mejor salud y reducción en la mortalidad cardiovascular (Avvenuti, Baiardini, y Giardini, 2016).

Respecto a el factor de amistad (estado de ánimo positivo de buena disposición hacia los demás), la presente submuestra da cuenta de una tendencia para generar recursos sociales importantes útiles para encarar los efectos adversos de la enfermedad y su recuperación (Affleck et al. 2001). Finalmente, en general, es de llamar la atención que en general los puntajes observados en las dimensiones anímicas negativas de la escala POMS para todas las muestras tienden a ser de moderados e incluso más altos que los reportados en la literatura internacional con enfermedades similares (e.g Baptista et al., 2017; Koizumi et al., 2018; McCorkle y Quint-Benoliel, 1983; Yang, Li y Liu, 2020).

Como limitaciones del estudio se pueden mencionar algunos aspectos empíricos de relevancia a considerar, sobresaliendo la necesidad de tomar en cuenta algunas variables clínicas relacionadas el desarrollo, curso y adaptación psicológica de los pacientes con respecto a su enfermedad (Okur et al., 2019). En primera instancia se desconoce el tiempo de diagnóstico y su relación con el desarrollo de algún tipo de malestar emocional asociado a la enfermedad, aspecto importante a 
considerar en posteriores investigaciones (e.g. Gonzalez-Zacarias et al., 2016). Asimismo, si bien en los pacientes con cáncer se consideró solamente la presencia de dolor y cronicidad, no obstante, no se tomaron en cuenta aquellos pacientes que no experimentaban dolor y su diferenciación con respecto a los IPE. De la misma manera, la inclusión de las experiencias de dolor (intensidad, grado de interferencia y la evaluación de sus dominios sensoriales, afectivos y cognitivos; Haribhakti, 2019) en el resto de los pacientes con otras ECnT no fue abordado, por lo que se aconseja que este tipo de mediciones contribuya en otras investigaciones afines a explicar de mejor manera las respuestas afectivas de dichos pacientes a su condición física.

Respecto a las repercusiones prácticas y sugerencias de investigaciones futuras, se considera que la investigación psicológica del perfil anímico de los pacientes con ECnT podría considerar algunos aspectos que en conjunto con el sexo de los pacientes podrían ser de relevancia, entre ellos el nivel de comparación específico de grupos de edad y la severidad e impacto discapacitante de la enfermedad (Fillingim, 2017). Dado que en la presente investigación se detectó que en algunos grupos no existieron diferencias en el perfil anímico en cuanto a las sub tipologías diagnósticas (e.g. pacientes con cáncer), se sugiere que investigaciones posteriores tomen en cuenta muestras de subgrupos diagnósticos más amplios y distribuidos de forma más homogénea con respecto a otras variables (e.g. tipo de tratamiento; Martins-Klein et al., 2019). Algunos datos abordados entre los grupos de ECnT plantean el reto de documentar de forma sistemática el seguimiento de las variaciones en el perfil anímico pre y post intervención médica y sus efectos a corto y largo plazo en la reducción de estados de ánimo negativos (Zhang, et al. 2019). Finalmente, los hallazgos de la presente investigación constituyen un llamado de atención importante para la atención a la salud mental de la población con enfermedades crónicas, buscando con ello proporcionar una mayor adherencia terapéutica, mejor calidad de vida y una adaptación saludable de las personas hacia su enfermedad. 


\section{Referencias}

Affleck, G., Tennen, H., Zautra, A., Urrows, S., Abeles, M., y Karoly, P. (2001). Women's pursuit of personal goals in daily life with fibromyalgia: A value-expectancy analysis. Journal of Consulting and Clinical Psychology, 69(4), 587-596. https://doi. org/10.1037/0022-006x.69.4.587

Akechi, T., Kugaya, A., Okamura, H., Yamawaki, S., y Uchitomi, Y. (1999). Fatigue and Its Associated Factors in Ambulatory Cancer Patients. Journal of Pain and Symptom Management, 17(1), 42-48. https://doi.org/10.1016/s0885-3924(98)00105-5

Allart-Vorelli, P., Porro, B., Baguet, F., Michel, A., y Cousson-Gélie, F. (2015). Haematological cancer and quality of life: a systematic literature review. Blood Cancer Journal, 5(4), e305-e305. https:// doi.org/10.1038/bcj.2015.29

Andrade, E., Arce, C., y Seoane, G. (2002). Adaptación al español del cuestionario Perfil de los Estados de Ánimo en una muestra de deportistas. Psicothema, 708-713.

Andreoulakis, E., Hyphantis, T., Kandylis, D., y Iacovides, A. (2012). Depression in diabetes mellitus: a comprehensive review. Hippokratia, 16(3), 205-214.

Andrew, M. J., Baker, R. A., Kneebone, A. C., y Knight, J. L. (2000). Mood state as a predictor of neuropsychological deficits following cardiac surgery. Journal of Psychosomatic Research, 48(6), 537-546. https://doi.org/10.1016/s0022-3999(00)00089-1

Annunziata, M. A., Muzzatti, B., Mella, S., y Bidoli, E. (2013). Fatigue, quality of life, and mood states during chemotherapy in Italian cancer patients. Tumori Journal, 99(1), 28-33. https:// doi.org/10.1177/030089161309900129

Arab, A., Mehrabani, S., Moradi, S., y Amani, R. (2018). The association between diet and mood: A systematic review of current literature. Psychiatry Research. https://doi.org/10.1016/j. psychres.2018.12.014 
Arpin, K., Fitch, M., Browne, G. B., y Corey, P. (1990). Prevalence and correlates of family dysfunction and poor adjustment to chronic illness in specialty clinics. Journal of Clinical Epidemiology, 43(4), 373-383. https://doi.org/10.1016/0895-4356(90)90123-7

Artom, M., Moss-Morris, R., Caskey, F., y Chilcot, J. (2014). Fatigue in advanced kidney disease. Kidney International, 86(3), 497-505. https://doi.org/10.1038/ki.2014.86

Atencio, B., Nucette, E., Colina, J., Sumalave, S., Gómez, F., y Hinestroza, D. (2004). Evaluación de la depresión y ansiedad en pacientes con insuficiencia renal crónica sometidos a hemodiálisis. Archivos Venezolanos de Psiquiatría y Neurología, 50, 35-41. Baptista, L. C., Machado-Rodrigues, A. M., y Martins, R. A. (2017). Exercise but not metformin improves health-related quality of life and mood states in older adults with type 2 diabetes. European Journal of Sport Science, 17(6), 794-804. https://doi.org/10 $.1080 / 17461391.2017 .1310933$

Barinková, K., y Mesároová, M. (2013). Anger, coping, and quality of life in female cancer patients. Social Behavior and Personality: An International Journal, 41(1), 135-142. https://doi. org/10.2224/sbp.2013.41.1.135

Bennett, P., Owen, R. L., Koutsakis, S., y Bisson, J. (2002). Personality, social context and cognitive predictors of post-traumatic stress disorder in myocardial infarction patients. Psychology \& Health, 17(4), 489-500. https://doi. org/10.1080/0887044022000004966

Breymeyer, K. L., Lampe, J. W., McGregor, B. A., y Neuhouser, M. L. (2016). Subjective mood and energy levels of healthy weight and overweight/obese healthy adults on high-and low-glycemic load experimental diets. Appetite, 107, 253-259. https://doi. org/10.1016/j.appet.2016.08.008

Brydon, L., Walker, C., Wawrzyniak, A. J., Chart, H., y Steptoe, A. (2009). Dispositional optimism and stress-induced changes in immunity and negative mood. Brain, Behavior, and Immunity, 23(6), 810-816. https://doi.org/10.1016/j.bbi.2009.02.018 
De Groot, M., Anderson, R., Freedland, K. E., Clouse, R. E., yLustman, P. J. (2001). Association of Depression and Diabetes Complications: A Meta-Analysis. Psychosomatic Medicine, 63(4), 619-630. https://doi.org/10.1097/00006842-200107000-00 015

Dupre, M. E., Beck, A. N., y Meadows, S. O. (2009). Marital trajectories and mortality among US adults. American Journal of Epidemiology, 170(5), 546-555. https://doi.org/10.1093/aje/ kwp 194

Elliott, P. C., Murphy, B. M., Oster, K. A., Le Grande, M. R., Higgins, R. O., y Worcester, M. U. C. (2010). Changes in mood states after coronary artery bypass graft surgery. European Journal of Cardiovascular Nursing, 9(3), 188-194. https://doi. org/10.1016/j.ejcnurse.2009.11.010

Evangelista, L. S., Moser, D. K., Westlake, C., Pike, N., Ter-Galstanyan, A., y Dracup, K. (2008). Correlates of fatigue in patients with heart failure. Progress in Cardiovascular Nursing, 23(1), 12-17. https://doi.org/10.1111/j.1751-7117.2008.07275.x

Fennessy, M. M., Fink, A. M., Eckhardt, A. L., Jones, J., Kruse, D. K., VanderZwan, K. J., Ryan, C. J., y Zerwic, J. J. (2010). Gender differences in fatigue associated with acute myocardial infarction. Journal of Cardiopulmonary Rehabilitation and Prevention, 30(4), 224-230.

Field, A. (2013). Discovering Statistics Using IBM SPSS Statistics. Londres: SAGE.

Fillingim, R. B. (2017). Sex, gender, and pain. Principles of GenderSpecific Medicine, 481-496. https://doi.org/10.1016/ b978-0-12-803506-1.00038-3

Fink, A. M., Eckhardt, A. L., Fennessy, M. M., Jones, J., Kruse, D., VanderZwan, K. J., ... Zerwic, J. J. (2010). Psychometric properties of three instruments to measure fatigue with myocardial infarction. Western Journal of Nursing Research, 32(7), 967-983. https://doi.org/10.1177/0193945910371320 
Fink, A. M., Sullivan, S. L., Zerwic, J. J., y Piano, M. R. (2009). Fatigue with systolic heart failure. The Journal of Cardiovascular Nursing, 24(5), 410-417. https://doi.org/10.1097/ jcn.0b013e3181ae1e84

Findıkl1, E., Camkurt, M.A., Izci, F., Yavuz, Y.C., Fındıkl, H.A., Altun, H., y Doğan, E. (2016). A case-control study on the affective temperament profiles, anxiety and depression levels of patients with chronic renal failure. Journal of Mood Disorders, 6(3), 133-139. https://doi.org/10.5455/jmood.20160321034554

Fritschi, C. y Quinn, L. (2010). Fatigue in patients with diabetes: a review. Journal of psychosomatic research, 69(1), 33-41.https:// doi.org/10.1016/j.jpsychores.2010.01.021

Giordano, M., Tirelli, P., Ciarambino, T., Gambardella, A., Ferrara, N., Signoriello, G., ... Varricchio, M. (2007). Screening of depressive symptoms in young-old hemodialysis patients: relationship between beck depression inventory and 15-item geriatric depression scale. Nephron Clinical Practice, 106(4), c187-c192. https:// doi.org/10.1159/000104430

Gonzalez-Zacarias, A. A., Mavarez-Martinez, A., Arias-Morales, C. E., Stoicea, N., y Rogers, B. (2016). Impact of Demographic, Socioeconomic, and Psychological Factors on Glycemic Self-Management in Adults with Type 2 Diabetes Mellitus. Frontiers in Public Health, 4, 195. https://doi.org/10.3389/ fpubh.2016.00195

Groarke, A., Curtis, R., Walsh, D. M. J., y Sullivan, F. J. (2018). What predicts emotional response in men awaiting prostate biopsy? BMC Urology, 18(1). https://doi.org/10.1186/ s12894-018-0340-9

Haribhakti V.V. (2019. Restoration, Reconstruction and Rehabilitation in Head and Neck Cancer. Singapore: Springer.

Helgeson, V. S. y Zajdel, M. (2017). Adjusting to chronic health conditions. Annual Review of Psychology, 68(1), 545-571. https:// doi.org/10.1146/annurev-psych-010416-044014 
Horne, D., Kehler, S., Kaoukis, G., Hiebert, B., Garcia, E., Duhamel, T. A., y Arora, R. C. (2013). Depression before and after cardiac surgery: Do all patients respond the same? The Journal of Thoracic and Cardiovascular Surgery, 145(5), 1400-1406. https:// doi.org/10.1016/j.jtcvs.2012.11.011

Ilie, G., Bradfield, J., Moodie, L., Lawen, T., Ilie, A., Lawen, Z., Blackman, C.,... Rutledge, R. (2019). The role of response-shift in studies assessing quality of life outcomes among cancer patients: A systematic review. Frontiers in Oncology, 9,783. https://doi. org/10.3389/fonc.2019.00783

Jensen, Ø., Bernklev, T., Gibbs, C., Moe, R. B., Hofsø, D., y JelsnessJørgensen, L.P. (2018). Fatigue in type 1 diabetes, prevalence, predictors and comparison with the background population. Diabetes Research and Clinical Practice, 143, 71-78. https://doi. org/10.1016/j.diabres.2018.06.012

Jiang, J., Li, Y., Shen, Q., Rong, X., Huang, X., Li, H., ... Tang, Y. (2018). Effect of pregabalin on radiotherapy-related neuropathic pain in patients with head and neck cancer: a randomized controlled trial. Journal of Clinical Oncology, 37(2), 135-143. https://doi.org/10.1200/jco.18.00896

Joshwa, B. y Campbell, M. (2017). Fatigue in Patients with Chronic Kidney Disease: Evidence and Measures. Nephrology Nursing Journal, 44(4), 337-343. https://doi.org/10.1038/ki.2014.86

Kao, Y.-Y., Lee, W.-C., Wang, R.-H., y Chen, J.-B. (2020). Correlation of sociodemographic profiles with psychological problems among hospitalized patients receiving unplanned hemodialysis. Renal Failure, 42(1), 255-262. https://doi.org/10.1080/0886 022x.2020.1736097

Keskin, G. y Engin, E. (2011). The evaluation of depression, suicidal ideation and coping strategies in haemodialysis patients with renal failure. Journal of Clinical Nursing, 20(19-20), 2721-2732. https://doi.org/10.1111/j.1365-2702.2010.03669.x

Kiecolt-Glaser, J. K., Page, G. G., Marucha, P. T., MacCallum, R. C., y Glaser, R. (1998). Psychological influences on surgical reco- 
very: Perspectives from psychoneuroimmunology. American Psychologist, 53(11), 1209-1218. https://doi.org/10.1037/0003066x.53.11.1209

Kim, Y., Hickok, J. T., y Morrow, G. (2006). Fatigue and Depression in Cancer Patients Undergoing Chemotherapy: An Emotion Approach. Journal of Pain and Symptom Management, 32(4), 311-321. https://doi.org/10.1016/j.jpainsymman.2006.05.007 Koenig, H. G. (1988). Depression in elderly hospitalized patients with medical illness. Archives of Internal Medicine, 148(9), 1929. https://doi.org/10.1001/archinte.1988.00380090031009

Koizumi, K., Tayama, J., Ishioka, T., Nakamura-Thomas, H., Suzuki, M., Hara, M., Makita, S. y Hamaguchi, T. (2018). Anxiety, fatigue, and attentional bias toward threat in patients with hematopoietic tumors. Plos One, 13(2), e0192056. https://doi. org/10.1371/journal.pone.0192056

Lane, R. (2014). Restoration of positive mood states in major depression as a potential drug development target. Journal of Psychopharmacology, 28(6), 527-535. https://doi. org/10.1177/0269881114532857

Lin, C-C., Lai, Y-L., y Ward, S. E. (2003). Effect of cancer pain on performance status, mood states, and level of hope among Taiwanese cancer patients. Journal of Pain and Symptom Management, 25(1), 29-37. https://doi.org/10.1016/S0885-3924(02)00542-0

Mantantzis, K., Schlaghecken, F., Sünram-Lea, S. I., y Maylor, E. A. (2019). Sugar rush or sugar crash? a meta-analysis of carbohydrate effects on mood. Neuroscience \& Biobehavioral Reviews, 101, 45-67. https://doi.org/10.1016/j.neubiorev.2019.03.016

Martins-Klein, B., Bamonti, P. A., Owsiany, M., Naik, A., y Moye, J. (2019). Age differences in cancer-related stress, spontaneous emotion regulation, and emotional distress. Aging \& Mental Health, 1-10. https://doi.org/10.1080/13607863.2019.1693 972 
Mbakwem, A., Aina, F., y Armadi, C. (2016). Depression in patients with heart failure: Is enough being done?. Cardiac Failure Review, 2(2),110-112. https://doi.org/10.15420/cfr.2016:21:1

McCorkle, R., y Quint-Benoliel, J. (1983). Symptom distress, current concerns and mood disturbance after diagnosis of life-threatening disease. Social Science \& Medicine, 17(7), 431-438. https:// doi.org/10.1016/0277-9536(83)90348-9

McFarland, M. J., Hayward, M. D., y Brown, D. (2013). I've got you under my skin: marital biography and biological risk. Journal of Marriage and Family, 75(2), 363-380. https://doi.org/10.1111/ jomf.12015

McNair, D.M., Lorr, M., y Droppleman, L.F. (1981). Manual for the profile of mood states. EdITS Educational and Industrial Testing Service.

McNair D. M., Lorr, M., y Droppelman L. F. (1992). Revised Manual for the Profile of Mood States. Educational and Industrial Testing Services.

McNair D. M, Lorr, M., y Droppleman, L.F. (1971). EITS manual for the Profile of Mood States. Educational and Industrial Testing Service.

Moe, G. (2016). Heart failure with multiple comorbidities. Current Opinion in Cardiology, 31(2), 209-216. https://doi.org/10.1097/ hco.0000000000000257

Neupane, I., Arora, R. C., y Rudolph, J. L. (2017). Cardiac surgery as a stressor and the response of the vulnerable older adult. Experimental Gerontology, 87, 168-174. https://doi.org/10.1016/j. exger.2016.04.019

Nikbakhsh, N., Moudi, S., Abbasian, S., y Khafri, S. (2014). Prevalence of depression and anxiety among cancer patients. Caspian Journal of Internal Medicine, 5(3), 167-170.

Nouwen, A., Winkley, K., Twisk, J., Lloyd, C. E., Peyrot, M., ... Pouwer, F. (2010). Type 2 diabetes mellitus as a risk factor for the onset of depression: a systematic review and meta-analysis. 
Diabetologia, 53(12), 2480-2486. https://doi.org/10.1007/ s00125-010-1874-x

Nowak, Z. y Laudański, K. (2014). The perception of the illness with subsequent outcome measure in more favorable in continuous peritoneal dialysis vs hemodialysis in the framework of appraisal model of stress. International Journal of Medical Sciences, 11(3), 291-297. https://doi.org/10.7150/ijms.5431

OMS (2018). Enfermedades no transmisibles. Recuperado de https://www.who.int/es/news-room/fact-sheets/detail/ noncommunicable-diseases

Okur, Z. E., Sattel, H., Witthöft, M., y Henningsen, P. (2019). Emotion regulation in patients with somatic symptom and related disorders: A systematic review. PloS one, 14(6), e0217277. https://doi.org/10.1371/journal.pone.0217277

Ozdemir, P. G., Selvi, Y., Boysan, M., Ozdemir, M., Akdağ, S., y Ozturk, F. (2015). Relationships between coronary angiography, mood, anxiety and insomnia. Psychiatry Research, 228(3), 355-362. https://doi.org/10.1016/j.psychres.2015.05.084

Páez, A., Jofré, M., Azpiroz, C., y Bortoli, M. (2009). Ansiedad y depresión en pacientes con insufciencia renal crónica en tratamiento de diálisis. Universitas Psychologica, 8(1), 117-124.

Palinkas, L. A., Wingard, D. L., y Barrett-Connor, E. (1990). Chronic illness and depressive symptoms in the elderly: a populationbased study. Journal of Clinical Epidemiology, 43(11), 1131-1141. https://doi.org/10.1016/0895-4356(90)90014-g

Paukert, A. L., LeMaire, A., y Cully, J. A. (2009). Predictors of depressive symptoms in older veterans with heart failure. Aging \& Mental Health, 13(4), 601-610. https://doi. org/10.1080/13607860802459823

Penninx, B., Beekman, A., Ormel, J., Kriegsman, D., Boeke, A., Van Eijk, J., y Deeg, D. (1996). Psychological status among elderly people with chronic diseases: Does type of disease play a part? Journal of Psychosomatic Research, 40(5), 521-534. https:// doi.org/10.1016/0022-3999(95)00620-6 
Perales-Montilla, C., Duschek, S., y Reyes del Paso, G. (2013). Influencia de los factores emocionales sobre el informe de síntomas somáticos en pacientes en hemodiálisis crónica: relevancia de la ansiedad. Nefrologia, 33, 816-25.

Perales-Montilla, C., García-León, A, y Reyes-del Paso, G. A. (2012). Psychosocial predictors of the quality of life of chronic renal failure patients undergoing haemodialysis. Nefrologia, 32(5), 622-630. https://dx.doi.org/10.3265/Nefrologia.pre2012. Jun. 11447

Roberts, M. C., y Ilardi S. S. (2003). Handbook of Research Methods in Clinical Psychology. Blackwell Publishing.

Rodrigue, J. R., Mandelbrot, D. A., Hanto, D. W., Johnson, S. R., Karp, S. J., y Pavlakis, M. (2010). A cross-sectional study of fatigue and sleep quality before and after kidney transplantation. Clinical Transplantation, 25(1), E13-E21. https://doi. org/10.1111/j.1399-0012.2010.01326.x

Rometsch, S., Greutmann, M., Latal, B., Bernaschina, I., Knirsch, W., Schaefer, C., ... Landolt, M. A. (2018). Predictors of quality of life in young adults with congenital heart disease. European Heart Journal - Quality of Care and Clinical Outcomes. https:// doi.org/10.1093/ehjqcco/qcy046

Rosenthal, R., y DiMatteo, M. R. (2001). Meta-Analysis: Recent Developments in Quantitative Methods for Literature Reviews. Annual Review of Psychology, 52(1), 59-82. https://doi. org/10.1146/annurev.psych.52.1.59

Rotella, F., Mannucci, E., (2013). Depression as a risk factor for diabetes: a meta-analysis of longitudinal studies. Journal of Clinical Psychiatry, 74, 31-37. https://doi.org/10.4088/JCP.12r07922

Sasai, T., y Onishi, C. (2017). Relationship between Uncertainty and Emotions in advanced lung cancer patients after initial therapy. The Journal of Medical Investigation, 64(1.2), 96-100. https:// doi.org/10.2152/jmi.64.96

Schneiderman, N., Antoni, M., Saab, P., y Ironson, G. (2001). Health Psychology: Psychosocial and Biobehavioral Aspects of Chronic 
Disease Management. Annual Review of Psychology, 52, 555-580. https://doi.org/10.1146/annurev.psych.52.1.555

Shin, N., Hill-Briggs, F., Langan, S., Payne, J. L., Lyketsos, C., yGolden, S. H. (2017). The association of minor and major depression with health problem-solving and diabetes self-care activities in a clinic-based population of adults with type 2 diabetes mellitus. Journal of Diabetes and Its Complications, 31(5), 880-885. https://doi.org/10.1016/j.jdiacomp.2017.01.026

Smith, E. (2002). When Culture and Biology Collide: Why We are Stressed, Depressed, and Self-obsessed. Rutgers University Press.

Sprangers, M. A., y Schwartz, C. E. (1999). Integrating response shift into health-related quality of life research: a theoretical model. Social Science \& Medicine, 48(11), 1507-1515. https://doi. org/10.1016/s0277-9536(99)00045-3

Stanton, A. L., Revenson, T. A., y Tennen, H. (2007). Health Psychology: Psychological Adjustment to Chronic Disease. Annual Review of Psychology, 58(1), 565-592. https://doi.org/10.1146/ annurev.psych.58.110405.085615

Tamura, Y., Urawa, A., Watanabe, S., Hasegawa, T., Ogura, T., Nishikawa, K., ... Okada, M. (2018). Mood status and quality of life in kidney recipients after transplantation. Transplantation Proceedings. https://doi.org/10.1016/j.transproceed.2018.03.077

Vodermaier, A., Linden, W., MacKenzie, R., Greig, D., y Marshall, C. (2011). Disease stage predicts post-diagnosis anxiety and depression only in some types of cancer. British Journal of Cancer, 105(12), 1814-1817. https://doi.org/10.1038/bjc.2011.503

Wang, R. C., Wang, S. J., Chang, Y. C., y Lin, C. C. (2007). Mood state and quality of sleep in cancer pain patients: a comparison to chronic daily headache. Journal of Pain and Symptom Management, 33(1), 32-39. https://doi.org/10.1016/j. jpainsymman.2006.06.013

Williams, A.G., Crane, P.B., y Kring, D. (2007). Fatigue in African American women on hemodialysis. Nephrology Nursing Journal, 34(6), 610-617. 
Wulff-Burchfield, E., Dietrich, M. S., Ridner, S., y Murphy, B. A. (2018). Late systemic symptoms in head and neck cancer survivors. Supportive Care in Cancer. https://doi.org/10.1007/ s00520-018-4577-3

Yang, X., Li, H. y Liu, B. (2020). Analysis on the application value of responsibility system holistic nursing in patients with renal insufficiency after hemodialysis. International Journal of Clinical and Experimental Medicine, 13(2), 875-883.

Zabora, J., Brintzenhofeszoc, K., Curbow, B., Hooker, C., y Piantadosi, S. (2001). The prevalence of psychological distress by cancer site. Psycho-Oncology, 10(1), 19-28. https://doi.org/10.1002/1 099-1611(200101/02)10:1<19::AID-PON501>3.0.CO;2-6

Zhang, A. Y., Ganocy, S., Fu, A. Z., Kresevic, D., Ponsky, L., Strauss, G., Bodner, D. R., y Zhu, H. (2019). Mood outcomes of a behavioral treatment for urinary incontinence in prostate cancer survivors. Supportive Care in Cancer, 27(12), 4461-4467. https://doi.org/10.1007/s00520-019-04745-w

Recibido: 2020-06-16

Revisado: 2021-11-02

Aceptado: 2021-11-18 